\title{
Accelerometry-Based Classification of Human Activities Using Markov Modeling
}

\author{
Andrea Mannini and Angelo Maria Sabatini \\ Scuola Superiore Sant' Anna, Piazza dei Martiri della Libertà 33, Pisa 56125, Italy \\ Correspondence should be addressed to Andrea Mannini, a.mannini@sssup.it \\ Received 25 February 2011; Revised 17 May 2011; Accepted 29 June 2011 \\ Academic Editor: Fabio Babiloni
}

Copyright ( $) 2011$ A. Mannini and A. M. Sabatini. This is an open access article distributed under the Creative Commons Attribution License, which permits unrestricted use, distribution, and reproduction in any medium, provided the original work is properly cited.

\begin{abstract}
Accelerometers are a popular choice as body-motion sensors: the reason is partly in their capability of extracting information that is useful for automatically inferring the physical activity in which the human subject is involved, beside their role in feeding biomechanical parameters estimators. Automatic classification of human physical activities is highly attractive for pervasive computing systems, whereas contextual awareness may ease the human-machine interaction, and in biomedicine, whereas wearable sensor systems are proposed for long-term monitoring. This paper is concerned with the machine learning algorithms needed to perform the classification task. Hidden Markov Model (HMM) classifiers are studied by contrasting them with Gaussian Mixture Model (GMM) classifiers. HMMs incorporate the statistical information available on movement dynamics into the classification process, without discarding the time history of previous outcomes as GMMs do. An example of the benefits of the obtained statistical leverage is illustrated and discussed by analyzing two datasets of accelerometer time series.
\end{abstract}

\section{Introduction}

Many technical applications could greatly benefit from the availability of systems that are capable of automatically classifying specific physical activities of human beings. In this paper, either static posture, for example, standing, or dynamic motion, for example, walking, is included in the term physical activity. The sort of contextual awareness coming from this knowledge [1] may help improving the performance of healthcare monitoring devices or promoting the development of advanced human-machine interfaces. In fact, the precise activity performed by the subject helps defining the context in which further estimation can be conducted. Consider, for instance, the problem of estimating the metabolic energy expenditure of a human subject by indirect methods [2]: these methods are reported to incur severe estimation errors in the absence of any information about the particular functional task in which the subject is actually involved $[2,3]$. In robotics, several applications, notably in rehabilitation engineering, demand some capability by the robot controller of recognizing the user's intent. In particular, smart walking support systems have been developed to assist motor-impaired persons and elderly in their efforts to stand and to walk $[4,5]$ or to detect gait instabilities of the user $[6,7]$ and minimize the risk of fall [7].

In principle, the wearable sensors needed to elicit the contextual information would be characterized by low power consumption, small size and weight, and adequate metrological specifications. Microelectromechanical systems (MEMS) motion sensors appear well matched to these requirements [8]. The methods investigated in this paper revolve around the processing of acceleration signals acquired from small networks of MEMS accelerometers positioned at few anatomical points of the human body. The machine learning algorithms considered in this paper are a useful complement to the computational methods that are used for pose estimation and navigation using inertial sensors [9].

A major part of this paper consists of illustrating and discussing an approach for classification of human physical activities, which is based on using Hidden Markov Models (HMMs). In principle, this approach aims at exploiting the information available on the movement dynamics, namely, the capability of recognizing activities performed 
at the current time is related to the classification outcomes provided in the past by the classifier. Accordingly, we talk about sequential classifiers, which differ from the socalled single-frame classifiers in the sense that the latter are interested in single activity primitives, in other words, elementary activities are studied in isolation from the history of previously detected activities [9-16].

Nowadays, HMMs find applications in a large number of recognition problems, including, but not limited to, speech recognition [17], hand gesture and sign language recognition [18], and controlling robotic tools by hand gesture [19]. Concerning the human activity recognition, most studies on the application of HMMs $[20,21]$ are based on camera recordings, as shown by Yamato et al. in [22], although few research reports are now aiming at applications of HMMs to collect data from wearable sensor systems [23, 24]. These studies focus on the validation of statistical models of each considered activity. In a different way, our approach is based on using lightweight wearable sensors and is oriented to exploit HMMs at a higher level. In particular, their use can be oriented towards modeling time relations between elements of a sequence of activities. Few applications of HMMs are reported in the literature as for the problem of classifying human physical activities from inertial sensors, probably because HMMs are known to be potentially plagued by severe difficulties of parameter estimation. In this paper we propose a way of alleviating this difficulty by adopting a supervised approach to classifier training. This approach is feasible when the data available in the training set are annotated.

\section{Materials and Methods}

2.1. Datasets for Physical Activity Classification. The present work is partly based on analyzing the dataset of acceleration waveforms published by Bao and Intille [10]; the dataset, henceforth referred to as the B\&I dataset, was released to us by the authors. Acceleration data, sampled at $76.25 \mathrm{~Hz}$, are acquired from five biaxial accelerometers, located at the hip, wrist, arm, ankle, and thigh. The original protocol is based on testing 20 subjects, who are requested to perform 20 activities of daily living. These include activities that primarily involve the use of the upper limbs (e.g., brushing teeth, scrubbing, folding laundry, eating or drinking, and working on computer), whole-body activities (e.g., stretching, and vacuuming), and activities that primarily involve the use of the lower limbs (e.g., walking, climbing stairs, running, and cycling). In addition, static postures are considered (e.g., standing still, sitting and relaxing, lying down and relaxing) together with quasi-static postures (e.g., reading, watching TV). The B\&I dataset include, for each subject, acceleration time series that are known to correspond to a specific activity performed by her/him. We refer to any activity as an activity primitive, to distinguish it from higherlevel activities, namely, motor behaviors that result from chaining several activity primitives in some temporal order. Acceleration signals related to higher-level activities are not available in the B\&I dataset, since the research goal in [10] is exclusively to test single-frame classifiers.

In this paper, we select the seven activities shown in Figure 1, giving rise to a reduced dataset, henceforth called seven-activity dataset. We assume that a sequence of activity primitives, say, an activity at the motor sentence level can be modeled using a first-order Markov chain, composed of a finite number $Q$ of states $S_{i}(Q=7)$. Each state accounts for an activity primitive, say, an elementary activity at the motor word level: $S_{1}$, lying down and relaxing; $S_{2}$, cycling; $S_{3}$, climbing stairs; $S_{4}$, walking; $S_{5}$, running; $S_{6}$, sitting and relaxing; $S_{7}$, standing still. The time evolution of a firstorder Markov chain is governed by the vector $\pi$ of prior probabilities and the transition probability matrix (TPM) A, which helps describing human actions at the behavioral level. The prior probability vector $\pi$, with size $(1 \times Q)$, is composed of the probabilities $\pi_{i}$ of each state $S_{i}$ of being the state $X$ at the initial time $t_{0}$

$$
\pi_{i}=\operatorname{Pr}\left(X\left(t_{0}\right)=S_{i}\right), \quad i=1, \ldots, Q
$$

The elements $a_{i j}$ of the matrix A, with size $(Q \times Q)$, are the probabilities of transitions from the state $S_{i}$ at time $t_{n}$ to the state $S_{j}$ occupied at time $t_{n+1}$

$$
a_{i j}=\operatorname{Pr}\left(X\left(t_{n}\right)=S_{j} \mid X\left(t_{n-1}\right)=S_{i}\right), \quad i, j=1, \ldots, Q .
$$

The prior and transition probabilities needed to create the Observable Markov Model (OMM) $(\boldsymbol{\pi}, \mathbf{A})$ associated with the Markov chain can be empirically determined based on observations of the activity behavior of a subject.

In order to overcome the limitations of the B\&I dataset when applied to model sequential data, we propose the concept of the virtual experiment. The virtual experiment is a sort of generative model, which allows to concatenate activities together and to simulate complex behaviors mainly for algorithm validation and testing. Simulating a complex behavior by a single subject in our study (virtual experiment) requires that each model state may emit data frames that are randomly sampled (with replacement) from the $N$ frames available for the given subject and the activity primitive associated to the emitting state $(18 \leq N \leq 58)$. Henceforth, data frames are referred to as sliding windows with finite and constant width, whose samples are used to compute the feature vectors needed by the classification algorithm, see Section 2.2. for the seven-activity dataset, the data frames last about 6.7 s (50\% overlapping). For each subject, we perform twenty virtual experiments $(S=20)$, each of which is composed of $T=300$ data frames. The OMM associated with each virtual experiment is built using the TPM specification shown in Table 1.

The procedure of synthesizing virtual experiments in the manner described above implies the existence of clearcut borders between data frames associated to different primitives, without transients between consecutive data frames that may be unknown to the classifier. This problem is usually managed by manual data cropping in creating the dataset [10]. Of course, real-life data would be more complex 
TABLE 1: TPM for the virtual experiments simulated in this paper (seven-activity dataset).

\begin{tabular}{|c|c|c|c|c|c|c|c|}
\hline & $S_{1}$ & $S_{2}$ & $S_{3}$ & $S_{4}$ & $S_{5}$ & $S_{6}$ & $S_{7}$ \\
\hline$S_{1}$ & 0.95 & 0.20 & 0.00 & 0.00 & 0.00 & 0.01 & 0.04 \\
\hline$S_{2}$ & 0.00 & 0.90 & 0.00 & 0.04 & 0.00 & 0.01 & 0.05 \\
\hline$S_{3}$ & 0.00 & 0.00 & 0.62 & 0.25 & 0.01 & 0.02 & 0.10 \\
\hline$S_{4}$ & 0.00 & 0.01 & 0.03 & 0.80 & 0.02 & 0.07 & 0.07 \\
\hline$S_{5}$ & 0.00 & 0.01 & 0.01 & 0.35 & 0.40 & 0.01 & 0.22 \\
\hline$S_{6}$ & 0.02 & 0.00 & 0.00 & 0.04 & 0.00 & 0.85 & 0.09 \\
\hline$S_{7}$ & 0.01 & 0.03 & 0.01 & 0.18 & 0.03 & 0.12 & 0.62 \\
\hline
\end{tabular}

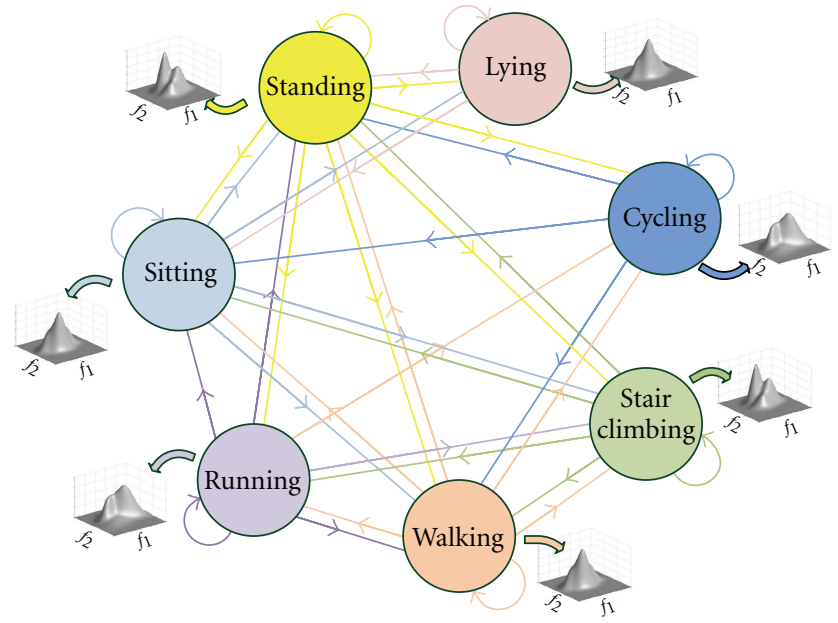

FIGURE 1: Scheme of a sequential classification based on HMMs.

and fuzzy, especially for the postural transitions between different activities. In the attempt to get a more realistic picture of the performance achieved by sequential classifier, data frames from the original dataset not included in the reduced dataset are randomly interspersed in the tested data sequences generated by the OMM in proportion $1: 3$. The resulting garbage is managed by the method of rejection of spurious data described in Section 2.5.

The virtual experiment approach we propose is to be considered a useful method for preliminary algorithm validation and testing. At the time being, the wearable system ActiNav is making its first steps in our lab for applications in the field of pedestrian navigation and smart estimation of biomechanical parameters; therefore, it is a welcome addition to the tools we have for investigating the Markov modeling approach to human activity classification.

ActiNav revolves around an ARMadeus Board (APF27). It is equipped with an ARM9-based Freescale processor, having $128 \mathrm{MB}$ of RAM, $256 \mathrm{MB}$ of flash memory, and a 200 K-gates Xilinx FPGA. A custom printed circuit board allows arming the APF27 with a 12-bit Successive Approximation Register ADC (AD7490, Analog Devices, Inc.). This converter operates up to 1 MSPS; moreover, since it is endowed with 16 analog channels, up to five triaxis analog accelerometers or gyros can be integrated in ActiNav. The system with the main board $(100 \times 84 \times 16 \mathrm{~mm})$ and different sensors connected is shown in Figure 2.

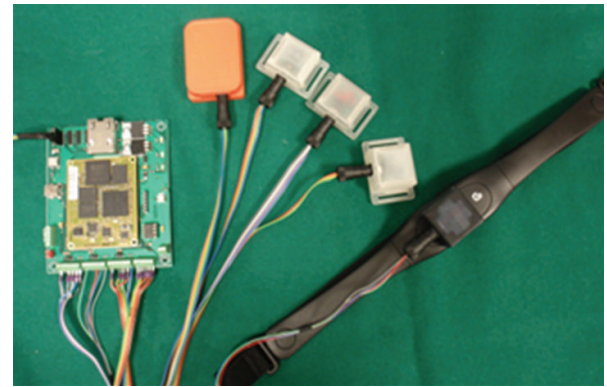

Figure 2: The ActiNav board is shown with several sensors connected to its input ports.

For the work described in this paper, a single triaxis accelerometer (ADXL325, Analog Devices, Inc.) with fullscale $\pm 5 \mathrm{~g}\left(1 \mathrm{~g}=9.81 \mathrm{~m} / \mathrm{s}^{2}\right)$ is fastened on the right thigh of seven healthy subjects. Each subject performed twenty repetitions of the following task: initially, he sat on a chair, then he stood up, and, after a pause of few seconds, he began to walk. Each trial lasted about $15 \mathrm{~s}$. Sensor data are acquired at a sampling frequency of $250 \mathrm{~Hz}$; moreover, they are manually annotated during acquisition (supervised approach). Specifically, the experimenter attempts to also define the time intervals where the transitions between different activities take place. The data frames are selected to last $250 \mathrm{~ms}$ (50\% overlapping). This low-complexity dataset, henceforth called the sit-stand-walk dataset, allows us to test the proposed methods on a real sequential dataset that includes a postural transition and the incipient locomotion situation.

2.2. Data Processing: Feature Vectors. The automatic classification of acceleration data requires a preprocessing phase in which feature variables with high information content are extracted from the data frames that the measurement channels of the system make available we have ten measurement channels in the seven-activity dataset and three measurement channels in the sit-stand-walk dataset.

Following the indications reported in previous works $[10,15]$, the feature variables considered in this paper are:

(i) DC component. This feature-helpful in discriminating static postures-is evaluated by averaging the raw samples in each data frame. One feature per measurement channel is obtained. 
(ii) Energy. This feature-helpful in assessing the activity strength-is evaluated as the sum of squared spectrogram coefficients within each data frame. The first coefficient that includes information about the DC component is excluded from the sum. One feature per measurement channel is obtained.

(iii) Entropy of spectrogram coefficients. This feature is helpful in discriminating activity primitives that differ in frequency domain complexity [10]. A kernel density estimator is applied to spectrogram coefficients for its determination. One feature per measurement channel is obtained.

(iv) Correlation coefficients. These features are the elements of the data covariance matrix; they are computed by applying the dot product to data frames from pairs of measurement channels, provided that the data frames are detrended and normalized to the window size; the correlation coefficients are helpful in discriminating activities that involve motions of various body parts. A total of 55 and 6 correlation coefficients are computed, respectively, for the sevenactivity and the sit-stand-walk datasets.

Before applying the classification algorithm, the feature vectors are selected in order to reduce the dimensionality of the problem, which can be critical especially for the sevenactivity dataset, where 85 feature variables are computed (15 for the sit-stand-walk dataset). Feature selection is required to limit the risk of incurring in severe overfitting [25]. We use the Pudil's algorithm-a sequential forward-backward floating search (SFFS-SFBS) [26]; this algorithm uses the Euclidean distances between each pair of feature vectors of the same class in the training set as a criterion for selecting the most informative feature variables. The criterion for optimizing the feature set derives from a cross-validation study based on a $k$-nearest neighbor classifier $(k-\mathrm{NN})$. Iteratively, the Pudil's algorithm modifies the number of features and repeats the validation process. After that, all features have been included once at least in the feature set the feature set of minimal size that maximizes the criterion is selected. A widely used feature extraction method, that is, the principal component analysis (PCA), is also applied to feature vectors [25].

2.3. Single-Frame Classification. Although several singleframe classifiers can be proposed, we consider here a particular technique for single-frame classification, namely, the Gaussian Mixture Model (GMM) classifier. This approach is reported by Allen et al. [9] to achieve very promising results. In particular, the authors discuss the high adaptability of the classifier, a good feature to analyze data from subjects that are not included in the training set.

Of course, other methods for single-frame classification of human physical activity can be chosen, and they may also outperform GMMs [27]. Here, the GMM classifier is selected as the single-frame classifier of reference, in particular for its resemblance to the structure of an HMM. As a matter of fact, the probability density of emissions of each state in an HMM can be modeled as a Gaussian mixture.

The GMM classifier first performs a parametric estimation of class-conditional probability density functions $p$ ( $\mathbf{x} \mid$ $w_{i}$ ), which assign the probabilities of the feature vector $\mathbf{x}$ given its membership to the class $w_{i}$. In the training phase, class-conditional probabilities are estimated as Gaussian mixtures. Each feature vector $\mathbf{x}$ is then classified in the class yielding the highest value of $p\left(\mathbf{x} \mid w_{i}\right)$.

2.4. HMM-Based Classification. In modeling sequences of human activities as first-order Markov chains, we propose that the prior and transition probabilities that are associated to the model are empirically determined by observing the subject behavior. If the TPM and the state at the current time are known, then the most likely state that will follow is probabilistically determined. However, each activity primitive can only be observed through a set of raw sensor signals (the measured time series from on-body accelerometers, in the present case). In other terms, the states are hidden and only a second-level process is actually observable (emissions). The statistical model including the pair $(\boldsymbol{\pi}, \mathbf{A})$ and the emission process is an HMM. We opt for a continuous emissions approach (continuous emissions densities HMM, aka cHMM, [17]). The most common approach to the problem of modeling continuous emissions is parametric. In particular we consider for the $i$-th state $S_{i}$, namely the class $w_{i}$, mixtures of $M$ multivariate normal distributions $N\left(\boldsymbol{\mu}_{i m}, \boldsymbol{\Sigma}_{i m}\right)$ that are specified by assigning the mean value vectors $\boldsymbol{\mu}_{i m}$, the covariance matrices $\boldsymbol{\Sigma}_{i m}$, and the matrix $\mathbf{C}$ of the mixing parameters $c_{i m}$ :

$$
p\left(\mathbf{x} \mid S_{i}\right)=\sum_{m=1}^{M} c_{i m} N\left(\mathbf{x} \mid \boldsymbol{\mu}_{i m}, \boldsymbol{\Sigma}_{i m}\right), \quad i=1, \ldots, Q,
$$

where

$$
\sum_{m=1}^{M} c_{i m}=1, \quad i=1, \ldots, Q
$$

The mixture is used to model the emissions from each state in the chain. An excellent reference source for HMMs and algorithms for their learning and testing in a recognition problem is in [17].

We consider a $Q$-state $\mathrm{CHMM}$ as represented in Figure 1 for the seven-activity dataset, where $Q=7$ (sit-standwalk dataset: $Q=3$ ). A Gaussian cHMM trained in a $d$ dimensional feature space, with $Q$ states and $M$ components for each mixture, requires the specification of the following parameters:

(i) $\pi$, prior probability vector, $1 \times Q$;

(ii) A, transition probability matrix $Q \times Q$;

(iii) $\boldsymbol{\mu}$, set of mean value matrices, $Q \times M \times d$;

(iv) $\boldsymbol{\Sigma}$, set of covariance matrices, $Q \times M \times d \times d$;

(v) C, set of mixing parameters, $Q \times M$.

One of the main problems with this approach may be in the high number of parameters to be identified. The 


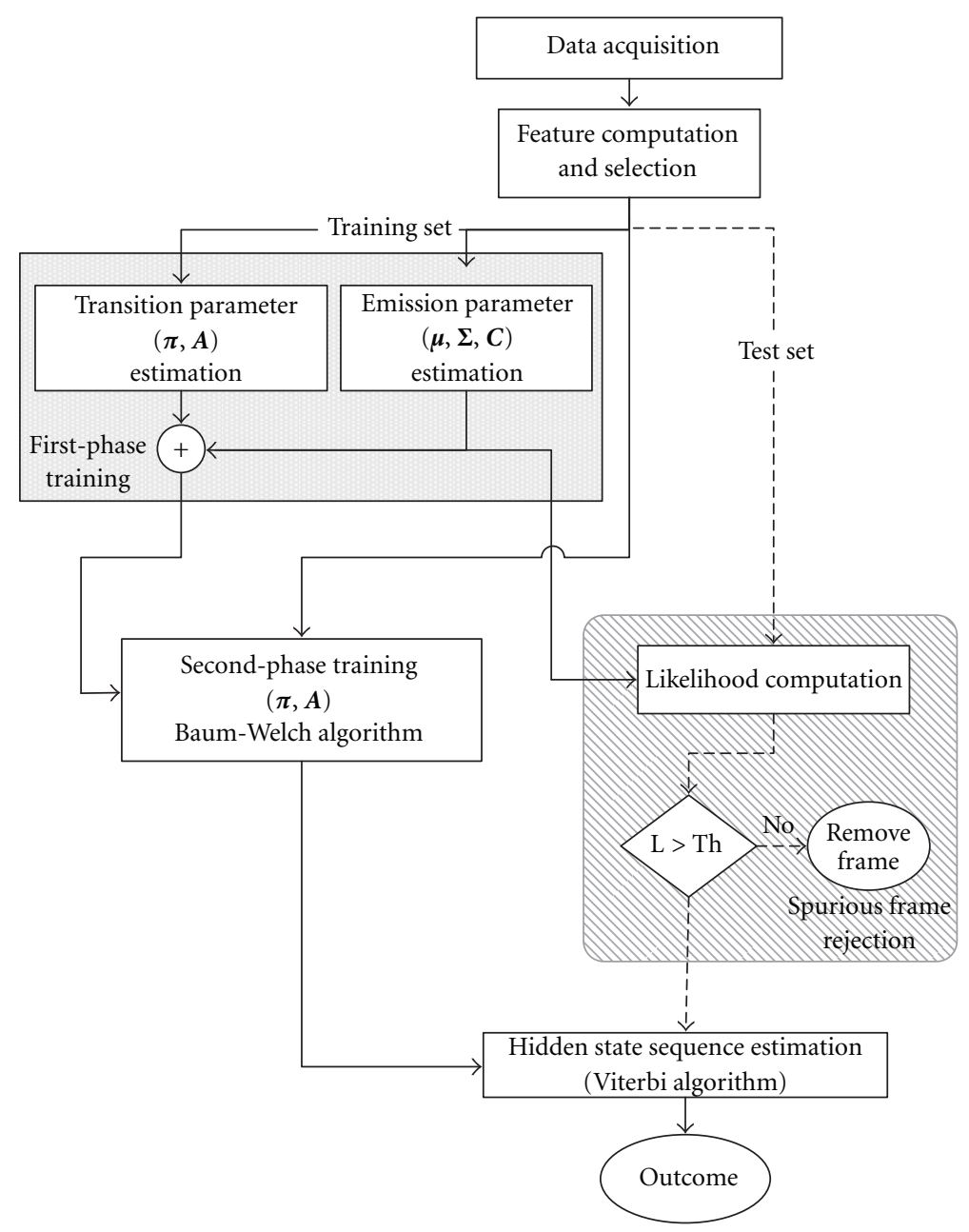

Figure 3: Block diagram of the developed cHMM-based sequential classifier.

approach to deal with the parameter identification problem we pursue is to split the training phase into two different steps: a first-level supervised training phase is followed by a second-level training phase, which is performed by running the Baum-Welch algorithm [17]. An inaccurate initialization of parameters could easily lead to suboptimal results when using the Baum-Welch algorithm, due to the presence of many local maxima in the optimization surface [17]. However, the training sets turn out to be labeled in the application described in this paper. Therefore, the first level supervised training becomes the proposed way for achieving a good initialization of parameters entering the second level of training.

In order to simplify the estimation process, the parameter set is divided into two main groups, namely, transition parameters $(\boldsymbol{\pi}, \mathbf{A})$ and emission parameters $(\boldsymbol{\mu}, \boldsymbol{\Sigma}, \mathbf{C})$. This separation allows us to train separately two parameter sets with reduced size, yielding a relevant reduction of the overall size of the training set. Since activity labels from training set examples are assumed to be known, simple methods of counting event occurrences allow us to estimate transition parameters [17]. For instance, the probability $a_{i j}$ of a transition from the $i$-the state to the $j$-th state is estimated as follows:

$$
\hat{a}_{i j}=\frac{N_{i \rightarrow j}}{N}
$$

where $N_{i \rightarrow j}$ is the number of transitions from the $i$-th state to the $j$-state counted in a training set with size $N$. Emission parameters can be estimated by fitting Gaussian mixture distributions with $M$ components to the feature vectors emitted by each state, in a similar fashion to the procedure used to learn GMM classifiers. The training process at the second level exploits the values of the parameters estimated during the training process at the first level, as initial values for running the Baum-Welch algorithm; in our current implementation, this step helps refining the estimates of the transition parameters, Figure 3. In the module for spurious frame rejection, the likelihood $\mathrm{L}$ is compared with a suitably chosen threshold Th. The optimal state sequence traced by the cHMM is estimated using a standard Viterbi decoder [17].

A leave-one-out validation study is performed in this paper for both GMM and cHMM-based classifiers. This 
means that a classifier is trained using data from all subjects but one, and then it is tested on data from the excluded subject only. The cross-validation process is repeated a number of times, each time excluding one different subject from training. Results are then aggregated from the different models. This validation approach allows testing the ability of each classifier to classify correctly new examples that differ from those used for training (generalization); good performances in terms of generalization are essential to prevent the need for individual model calibrations.

2.5. Spurious Data Rejection. The strategy of classification we adopt allows us to define a criterion for automatic rejection of spurious feature vectors. If a threshold-based detector is applied to estimated class-conditional probabilities $p(\mathbf{x} \mid$ $\left.w_{i}\right)$, it is possible to reject those feature vectors in the classification of which is believed to be too uncertain; we are not forced to introduce additional states, or mixture components, specifically for unknown data. Remind that, in a GMM or a cHMM, $p\left(\mathbf{x} \mid w_{i}\right)$ refers to the probability of the feature vector $\mathbf{x}$ as the emission of the model state $w_{i}$. If, for any feature vector, the probabilities relative to each state are below a given threshold, the feature vector itself can be marked as spurious and removed, without affecting the classifier operation. Low values of $p\left(\mathbf{x} \mid w_{i}\right)$ are typical when unknown activities are hidden in the data presented to the classifier or when too much uncertainty affects them.

The threshold value is optimized by studying the specificity-sensitivity curve (ROC curve); averaged across subjects, it is reported in Figure 4 (seven-activity dataset). The threshold is chosen when the specificity is slightly greater than the sensitivity.

\section{Results}

We empirically determine whether or not rotation and dimensionality reduction would be jointly pursued when the PCA is applied to the feature variables surviving the Pudil's method. Guided by the results of preliminary testing, we use the PCA cascaded to the Pudil's method for pure rotation in the feature space (seven-activity dataset) and for rotation and dimensionality reduction (sit-stand-walk dataset). In the former case, thirteen features_all of them being correlation coefficients-are retained for further processing. In the case of the sit-stand-walk dataset, three principal components are considered ( $97.8 \%$ of variance is retained), after that the Pudil's method selected seven out of fifteen feature variables.

The $k$-NN classification accuracies achieved by the Pudil's method are $99.5 \%$ and $99.2 \%$ (seven-activity and sitstand-walk dataset, resp.). It is important to outline that these values have nothing to do with the classification accuracies reported in the following for the GMM and the cHMMbased classifiers. Indeed, the feature selection process is based on a cross-validation study extended to the whole dataset, while classifier testing is based on a leave-one-out approach.

The number of Gaussian components of the mixture is taken $M=1$, both in the GMM and the cHMMbased classifiers. The experimental evidence does not clearly

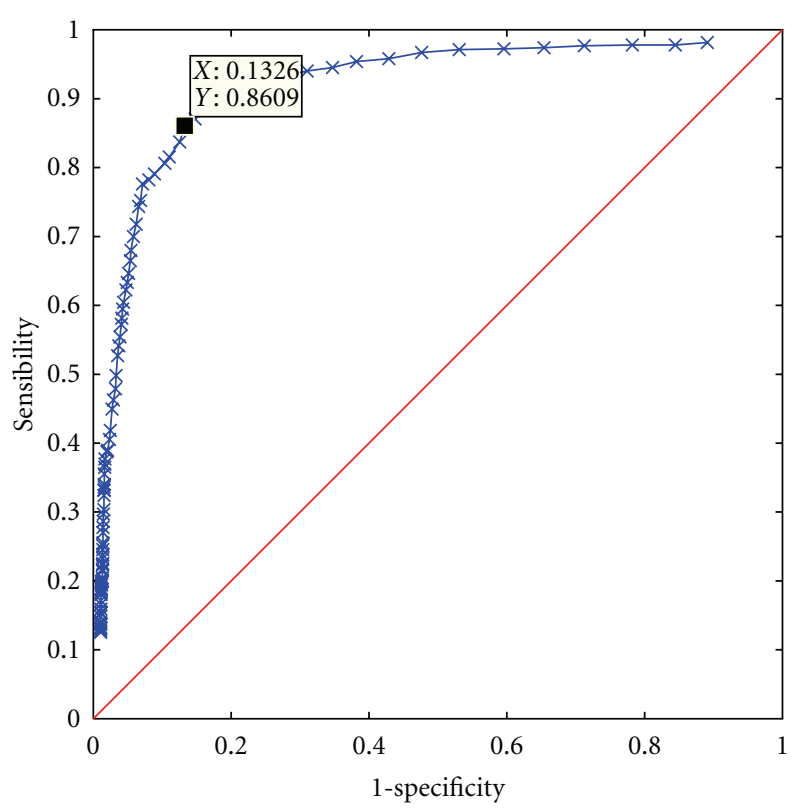

Figure 4: ROC curve obtained using different threshold values.

support the assumption that the data distributions are unimodal for both datasets; nonetheless, testing up to $M=5$ does not provide convincing arguments that the simpler choice $M=1$ would be dismissed. As for the seven-activity dataset, Tables 2 and 3 clearly show that $M=1$ is the winning choice in most cases. This is probably due to the higher number of parameters that need to be estimated when $M$ increases. As for the sit-stand-walk dataset, the results in Table 4 seem to indicate a preference for values of $M$ greater than one. However, this is only when the mechanism for rejecting spurious data is disabled; otherwise, $M=1$ is the winning choice. In the following we only present results in the case that $M=1$.

3.1. The Seven-Activity Dataset. In Table 3, the classification accuracy, averaged across all tested classifiers, is reported. The estimated TPM turned out to be practically identical to the TPM specified in Table 1 to define virtual experiments. As far as the algorithm for rejection of spurious data is concerned, the threshold is fixed so as to achieve sensitivity $(\mathrm{Se}=86.1 \%)$ and specificity $(\mathrm{Sp}=86.7 \%$.) The classification accuracy in the presence of spurious data and after their automatic rejection is presented for either the GMM or the cHMM. The confusion matrix obtained by HMM $(1 s t+2 n d$ level) classification after spurious data rejection is reported in Table 5 results from all subjects are aggregated.

For some unknown reasons, we observe that three subjects are characterized by unusually low values of classification accuracy. Table 6 shows the results when these subjects are not considered.

3.2. The Sit-Stand-Walk Dataset. The classification accuracy results are reported in Table 4 whereas the confusion matrix for the HMM classifier after rejection of spurious data is 
TABLE 2: Classification accuracy averaged over the twenty subjects available in the seven-activity dataset. Spurious data are not inserted. The values are reported as mean \pm standard deviation.

\begin{tabular}{|c|c|c|c|c|c|}
\hline \multicolumn{6}{|c|}{ Seven-activity dataset (in the absence of spurious data) } \\
\hline & $M=1$ & $M=2$ & $M=3$ & $M=4$ & $M=5$ \\
\hline GMM & $88.6 \pm 13.7$ & $88.1 \pm 13.4$ & $85.9 \pm 13.6$ & $82.5 \pm 16.4$ & $82.0 \pm 16.6$ \\
\hline cHMM (1st level) & $90.2 \pm 13.0$ & $88.0 \pm 12.9$ & $85.6 \pm 13.0$ & $80.8 \pm 17.1$ & $79.3 \pm 17.7$ \\
\hline cHMM (1st + 2nd level) & $90.2 \pm 13.0$ & $88.8 \pm 13.4$ & $85.2 \pm 14.5$ & $83.5 \pm 16.3$ & $82.8 \pm 18.1$ \\
\hline
\end{tabular}

TABLE 3: Classification accuracy averaged over the twenty subjects available in the seven-activity dataset in the presence of spurious data. The values are reported as mean \pm standard deviation.

\begin{tabular}{|c|c|c|c|c|c|}
\hline \multicolumn{6}{|c|}{ Seven-activity dataset (spurious data present in proportion $1: 3$ ) } \\
\hline & $M=1$ & $M=2$ & $M=3$ & $M=4$ & $M=5$ \\
\hline \multicolumn{6}{|c|}{ Without rejection of spurious data } \\
\hline GMM & $63.3 \pm 9.9$ & $62.4 \pm 11.4$ & $60.2 \pm 11.2$ & $60.5 \pm 13.0$ & $56.0 \pm 11.2$ \\
\hline cHMM (1st level) & $63.8 \pm 9.3$ & $61.4 \pm 10.6$ & $57.8 \pm 10.9$ & $55.0 \pm 14.0$ & $42.6 \pm 13.9$ \\
\hline $\mathrm{cHMM}(1 \mathrm{st}+2 \mathrm{nd}$ level $)$ & $63.8 \pm 9.3$ & $61.8 \pm 9.7$ & $57.0 \pm 12.5$ & $50.1 \pm 15.5$ & $43.4 \pm 14.6$ \\
\hline \multicolumn{6}{|l|}{ With rejection of spurious data } \\
\hline GMM & $85.4 \pm 13.4$ & $86.7 \pm 14.9$ & $83.4 \pm 15.3$ & $80.5 \pm 16.2$ & $81.7 \pm 17.6$ \\
\hline cHMM (1st level) & $86.2 \pm 12.9$ & $87.1 \pm 13.7$ & $83.0 \pm 14.6$ & $80.2 \pm 15.7$ & $81.9 \pm 17.6$ \\
\hline cHMM $(1 \mathrm{st}+2 \mathrm{nd}$ level $)$ & $86.2 \pm 12.8$ & $86.1 \pm 13.3$ & $83.2 \pm 14.0$ & $76.1 \pm 16.7$ & $75.9 \pm 20.6$ \\
\hline
\end{tabular}

TABLE 4: Classification accuracy averaged over the seven subjects available in the sit-stand-walk dataset. The values are reported as mean \pm standard deviation.

\begin{tabular}{|c|c|c|c|c|c|}
\hline \multicolumn{6}{|c|}{ Sit-stand-walk dataset } \\
\hline & $M=1$ & $M=2$ & $M=3$ & $M=4$ & $M=5$ \\
\hline \multicolumn{6}{|c|}{ Without rejection of spurious data } \\
\hline GMM & $94.1 \pm 1.8$ & $93.7 \pm 4.1$ & $94.8 \pm 2.4$ & $95.5 \pm 1.9$ & $95.8 \pm 2.1$ \\
\hline HMM (1st level) & $95.2 \pm 7.0$ & $96.8 \pm 3.0$ & $98.5 \pm 1.0$ & $98.3 \pm 1.1$ & $98.1 \pm 1.1$ \\
\hline HMM (1st + 2nd level) & $95.2 \pm 7.0$ & $97.4 \pm 2.9$ & $97.9 \pm 2.4$ & $97.0 \pm 2.9$ & $98.5 \pm 1.0$ \\
\hline \multicolumn{6}{|c|}{ With rejection of spurious data } \\
\hline GMM & $94.0 \pm 1.8$ & $95.6 \pm 1.5$ & $95.8 \pm 2.3$ & $95.6 \pm 1.7$ & $95.6 \pm 1.5$ \\
\hline HMM (1st level) & $99.0 \pm 1.0$ & $98.6 \pm 1.0$ & $98.8 \pm 1.0$ & $98.8 \pm 1.1$ & $98.6 \pm 1.1$ \\
\hline HMM (1st + 2nd level) & $99.0 \pm 1.0$ & $98.8 \pm 1.0$ & $98.8 \pm 1.0$ & $98.7 \pm 1.1$ & $98.7 \pm 1.0$ \\
\hline
\end{tabular}

TABLE 5: Confusion matrix obtained comparing HMM (1st +2 nd level) classifier output (columns) and the actual activity class labels after spurious data rejection (rows). All subjects' results are aggregated.

\begin{tabular}{|c|c|c|c|c|c|c|c|}
\hline $\mathrm{cHMM}$ & $S_{1}$ & $S_{2}$ & $S_{3}$ & $S_{4}$ & $S_{5}$ & $S_{6}$ & $S_{7}$ \\
\hline$S_{1}$ & 9686 & 10 & 3 & 29 & 1 & 53 & 22 \\
\hline$S_{2}$ & 0 & 6064 & 15 & 15 & 0 & 0 & 682 \\
\hline$S_{3}$ & 0 & 29 & 1825 & 2543 & 25 & 0 & 921 \\
\hline$S_{4}$ & 0 & 420 & 347 & 19189 & 35 & 0 & 613 \\
\hline$S_{5}$ & 0 & 2 & 4 & 1 & 580 & 6 & 23 \\
\hline$S_{6}$ & 2 & 0 & 0 & 0 & 0 & 18823 & 0 \\
\hline$S_{7}$ & 0 & 293 & 47 & 376 & 3 & 0 & 9939 \\
\hline
\end{tabular}


TABle 6: Classification accuracy averaged over the seventeen subjects available in the seven-activity dataset, after removal of three anomalous subjects (see text). Spurious data are not inserted and $M=1$.

\begin{tabular}{lc}
\hline Classifier & Accuracy \\
\hline GMM & $93.5 \pm 6.0$ \\
cHMM (1st level) & $95.1 \pm 4.8$ \\
cHMM (1st + 2nd level) & $95.1 \pm 4.8$ \\
\hline
\end{tabular}

TABle 7: Confusion matrix obtained comparing HMM (1st + 2nd level) classifier output (columns) and the actual activity class labels after spurious data rejection (rows). All subjects' results are aggregated.

\begin{tabular}{lccc}
\hline cHMM & Sit & Stand & Walk \\
\hline Sit & 4973 & 10 & 0 \\
Stand & 0 & 3989 & 28 \\
Walk & 0 & 64 & 2270 \\
\hline
\end{tabular}

reported in Table 7. The spurious rejection algorithm is now applied to tag data with low reliability for classification. A higher number of tagged data is close to where activity transitions take place, Figure 6. Finally, Figure 5 describes the action of the spurious data rejection mechanism more in detail. The upper plot shows a typical example of a bad behavior of the Viterbi decoder close to a sit-to-stand transition when the rejection mechanism is turned off. A sit-to-stand transition is immediately followed by a stand-towalk transition, which is wrong. Now, given that the cHMM emerges from training with a left-right structure, flying back to stand once the system enters walk is forbidden. As the lower plot shows, when the system decides not to decide in the presence of data frames of uncertain origin, the action of the Viterbi decoder may be more correct. This time, indeed, the sit-to-stand transition is correctly observed, and the system is ready to recognize the stand-to-walk quite close to when it actually occurs.

\section{Discussions and Conclusions}

Our decision to concentrate on a basic vocabulary of activities is motivated by our ongoing work aimed at developing a wearable sensor system for pedestrian navigation and human locomotion rehabilitation. Referring to the seven-activity dataset, the Pudil's feature selection scheme individuates a subset of features that simply consist of movement coordination information (correlation coefficients). Nonetheless, it is argued that DC component, energy, and entropy timedomain features would be highly valuable, provided that we decide to investigate other activities, for example, those from the set studied in [10] that are not considered in this paper. Although being limited to three activities chained in a fixed order, and lasting few seconds only, the tests on the sit-standwalk dataset show that the proposed algorithm can also be applied to data in which activity transitions are naturalistic.

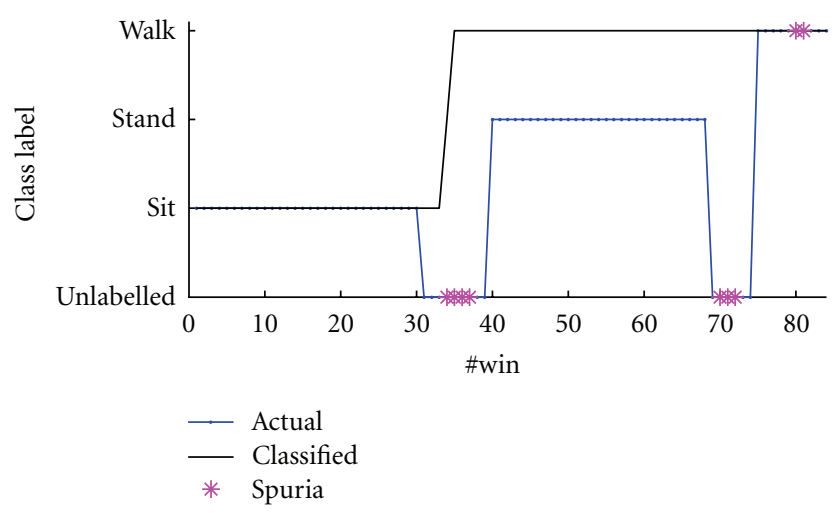

(a)

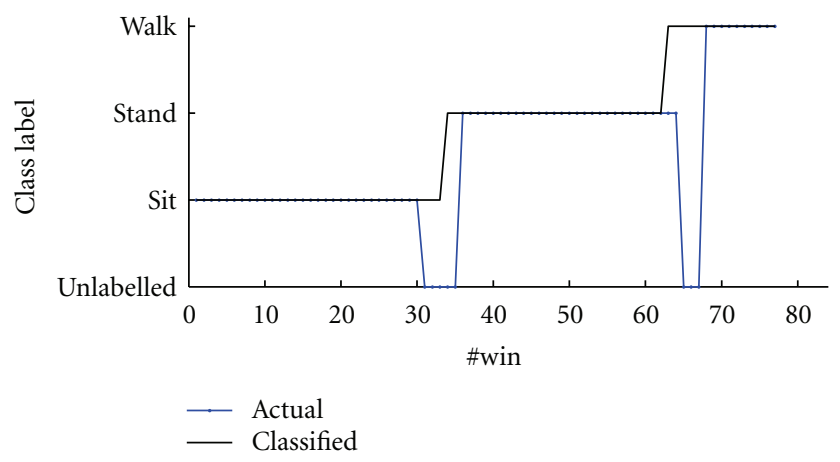

(b)

FIGURE 5: The method of spurious data rejection is shown in action (see text).

The generalization abilities of the tested classifiers can be considered good, since, for both datasets, a leave-oneout-subject validation strategy is adopted. Nonetheless, the classification accuracies are high, although the variability in terms of standard deviation is particularly high in the virtual experiments on the seven-activity dataset. This may be due to different factors: as compared with the sit-standwalk dataset, a higher number of activities and subjects are considered in the seven-activity dataset, and, finally, the data contamination policy in the virtual experiments is quite aggressive. The main fact is, we believe, that some subjects may perform the same activities differently with respect to other subjects: as our data in Tables 2 and 4 clearly show, this is at odds with the generalization ability of tested classifiers.

The supervised training is pursued in this paper with the idea to split the process of estimating the parameters of the cHMM-based classifier into two distinct levels. The secondlevel training process is based on parameter reestimation using the Baum-Welch algorithm. In the results reported in this paper, the effects of the Baum-Welch algorithm are uncertain and of limited utility. However, the BaumWelch algorithm is the classic approach for HMM parameter reestimation, even when a good initialization for supervised training is available $[23,28-31]$. The rationale for parameter reestimation in a supervised framework is the possible improvement of generalization capabilities by the classifier 


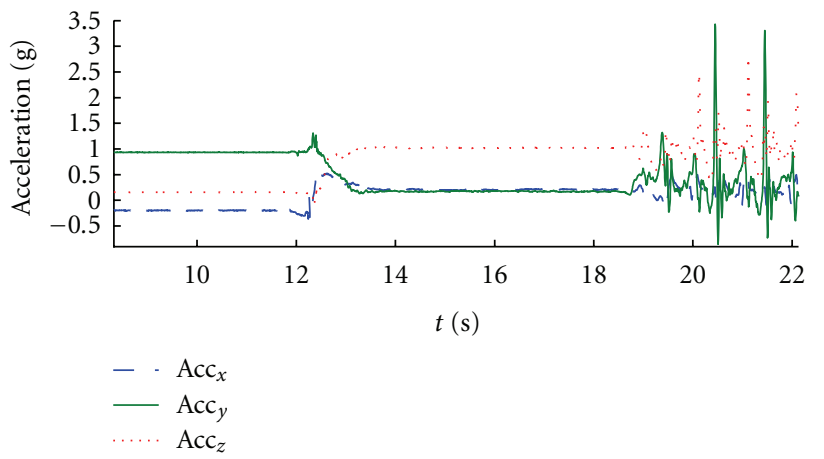

(a)

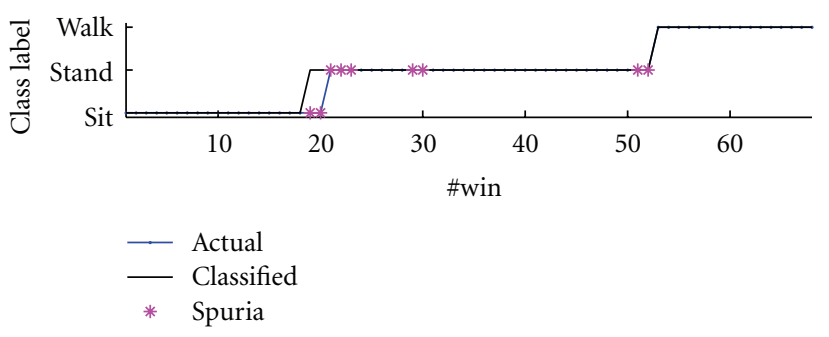

(b)

FIgure 6: Classification and spurious data rejection on a sequence of the sit-stand-walk dataset.

at the expense of some reduction of its specificity. Some recent studies discuss an interesting approach where the Baum-Welch algorithm is modified in the attempt to make an HMM-based classifier adaptive [32]. We are currently investigating a modification of our proposed classifiers in this direction.

An interesting point in our approach is related to the proposed method for managing spurious feature vectors. Most published studies, including [10], handle the problem of the fuzzy borders by manual data cropping. Clearly this is neither useful nor applicable if we look for a realtime system for activity classification. In our approach, the whole spurious rejection process is made automatic. When one-third of the whole feature vectors in the data are spurious, such as in the virtual experiments described in the paper, the classification accuracies of the tested sequential classifiers are limited to about $64 \%$ in the absence of the proposed threshold-based detector. If the threshold-based detector is actually implemented, the performances ramp up to about $86 \%$. When the spurious data cannot be tagged with certainty in advance, such as in the sit-standwalk experiments, the performance boost provided by the threshold-based detector is not as impressive as in the virtual experiments however, we see that the cHMM benefits from a quite remarkable increment from $95 \%$ to $99 \%$.

The cHMM-based classifier outperforms the GMM classifier by exploiting the statistical information of the activity dynamics. However, the statistical leverage of the HMM comes with its own problems. This is evident in analyzing the results obtained by working with the sit-stand-walk dataset. First, we observe that, in the sit-stand-walk experiments, on average, the performances of either the GMM or the cHMM are quite similar. However, in the absence of the thresholdbased detector of spurious data, the cHMM tends to exhibit a more erratic behavior as compared with the GMM, see results in Table 5. Why is this so? The cHMM relies on data that it has to be considered emitted from its internal states, in other words, the Markov model must account for all possible observations in order to draw meaningful inferences. When something goes wrong, the cHMM tends to be stubborn in relying on its statistical memory, which is however wrong, as vividly shown in Figure 5 . When a GMM classifier is considered, the emission models are the same as in the CHMM, but the GMM classifier does not pay regard to connections between states which are not modeled. Fortunately, when spurious data are prevented from affecting its behavior, the cHMM tends to perform better than the GMM classifier.

In conclusion, the applicability of Markovian modeling to the classification of human physical activities has been demonstrated. In particular, we have highlighted the importance of exploiting the statistical knowledge about the human motion dynamics that can be "trapped" within the Markov chain. The algorithm includes an effective device for rejecting spurious feature vectors, which turns out to show high sensitivity and specificity of detection.

\section{Acknowledgments}

The authors are indebted to Professor Stephen S. Intille, for allowing them to use his acceleration dataset for the computer experiments in this paper. They are also grateful to the anonymous reviewers for their valuable comments, which helped improving the quality of the paper.

\section{References}

[1] P. Brézillon, "Context in problem solving: a survey," Knowledge Engineering Review, vol. 14, no. 1, pp. 47-80, 1999.

[2] G. Meijer, K. R. Westerterp, F. M. Verhoeven, H. B. Koper, and F. ten Hoor, "Methods to assess physical activity with special reference to motion sensors and accelerometers," IEEE Transactions on Biomedical Engineering, vol. 38, no. 3, pp. 221229, 1991.

[3] C. V. Bouten, K. T. M. Koekkoek, M. Verduin, R. Kodde, and J. D. Janssen, "A triaxial accelerometer and portable data processing unit for the assessment of daily physical activity," IEEE Transactions on Biomedical Engineering, vol. 44, no. 3, pp. 136-147, 1997.

[4] H. Yu, M. Spenko, and S. Dubowsky, "An adaptive shared control system for an intelligent mobility aid for the elderly," Autonomous Robots, vol. 15, no. 1, pp. 53-66, 2003.

[5] O. Y. Chuy, Y. Hirata, and K. Kosuge, "A control approach based on passive behavior to enhance user interaction," IEEE Transactions on Robotics, vol. 23, no. 5, pp. 899-908, 2007.

[6] A. M. Sabatini, V. Genovese, and E. Pacchierotti, "A mobility aid for the support to walking and object transportation of people with motor impairments," in Proceedings of the IEEE/RSJ International Conference on Intelligent Robots and Systems, vol. 2, pp. 1349-1354, December 2002.

[7] Y. Hirata, S. Komatsuda, and K. Kosuge, "Fall prevention control of passive intelligent walker based on human model," 
in Proceedings of the IEEE/RSJ International Conference on Intelligent Robots and Systems, pp. 1222-1228, Nice, France, September 2008.

[8] A. M. Sabatini, "Inertial sensing in biomechanics: a survey of computational techniques bridging motion analysis and personal navigation," in Computational Intelligence for Movement Sciences: Neural Networks and Other Emerging Techniques, R. Begg and M. Palaniswami, Eds., pp. 70-100, Idea Group, Hershey, PA, USA, 2006.

[9] F. R. Allen, E. Ambikairajah, N. H. Lovell, and B. G. Celler, "Classification of a known sequence of motions and postures from accelerometry data using adapted Gaussian mixture models," Physiological Measurement, vol. 27, no. 10, pp. 935$951,2006$.

[10] L. Bao and S. S. Intille, "Activity recognition from userannotated acceleration data," Pervasive Computing, vol. 301, pp. 1-17, 2004.

[11] R. Begg and J. Kamruzzaman, "A machine learning approach for automated recognition of movement patterns using basic, kinetic and kinematic gait data," Journal of Biomechanics, vol. 38, no. 3, pp. 401-408, 2005.

[12] F. Foerster, "Detection of posture and motion by accelerometry: a validation study in ambulatory monitoring," Computers in Human Behavior, vol. 15, no. 5, pp. 571-583, 1999.

[13] D. M. Karantonis, M. R. Narayanan, M. Mathie, N. H. Lovell, and B. G. Celler, "Implementation of a real-time human movement classifier using a triaxial accelerometer for ambulatory monitoring," IEEE Transactions on Information Technology in Biomedicine, vol. 10, no. 1, pp. 156-167, 2006.

[14] M. J. Mathie, B. G. Celler, N. H. Lovell, and A. C. F. Coster, "Classification of basic daily movements using a triaxial accelerometer," Medical and Biological Engineering and Computing, vol. 42, no. 5, pp. 679-687, 2004.

[15] N. Ravi, N. Dandekar, P. Mysore, and M. L. Littman, "Activity recognition from accelerometer data," American Association for Artificial Intelligence, vol. 5, pp. 1541-1546, 2005.

[16] K. Van Laerhoven and O. Cakmakci, "What shall we teach our pants?" in Proceedings of the 4th Intenational Symposium on Wearable Computers, pp. 77-83, Atlanta, Ga, USA, October 2000.

[17] L. R. Rabiner, "Tutorial on hidden Markov models and selected applications in speech recognition," Proceedings of the IEEE, vol. 77, no. 2, pp. 257-286, February 1989.

[18] R. H. Liang and M. Ouhyoung, "A real-time continuous gesture recognition system for sign language," in Proceedings of the 3rd IEEE International Conference on Automatic and Gesture Recognition, pp. 558-567, Nara, Japan, April 1998.

[19] J. Yang, Y. Xu, and C. S. Chen, "Human action learning via hidden Markov model," IEEE Transactions on Systems, Man, and Cybernetics Part A: Systems and Humans, vol. 27, no. 1, pp. 34-44, 1997.

[20] R. Venkatesh Babu, B. Anantharaman, K. R. Ramakrishnan, and S. H. Srinivasan, "Compressed domain action classification using HMM," Pattern Recognition Letters, vol. 23, no. 10, pp. 1203-1213, 2002.

[21] F. Martinez-Contreras, C. Orrite-Urunuela, E. Herrero-Jaraba, H. Ragheb, and S. A. Velastin, "Recognizing human actions using silhouette-based HMM," in Proceedings of the 6th IEEE International Conference on Advanced Video and Signal Based Surveillance, pp. 43-48, Genova, Italy, September 2009.

[22] J. Yamato, J. Ohya, and K. Ishii, "Recognizing human action in time-sequential images using hidden Markov model," in Proceedings of the IEEE Computer Society Conference on Computer
Vision and Pattern Recognition, pp. 379-385, Yokosuka, Japan, 1992.

[23] D. M. Pober, J. Staudenmayer, C. Raphael, and P. S. Freedson, "Development of novel techniques to classify physical activity mode using accelerometers," Medicine and Science in Sports and Exercise, vol. 38, no. 9, pp. 1626-1634, 2006.

[24] S. Takeuchi, S. Tamura, and S. Hayamizu, "Human action recognition using acceleration information based on hidden Markov model," in Proceedings of the Asia-Pacific Signal and Information Processing Association, Annual Summit and Conference, Annual Summit and Conference (APSIPA ASC '09), pp. 829-832, Sapporo, Japan, October 2009.

[25] A. K. Jain, P. W. Duin, and J. Mao, "Statistical pattern recognition: a review," IEEE Transactions on Pattern Analysis and Machine Intelligence, vol. 22, no. 1, pp. 4-37, 2000.

[26] P. Pudil, J. Novovičová, and J. Kittler, "Floating search methods in feature selection," Pattern Recognition Letters, vol. 15, no. 11, pp. 1119-1125, 1994.

[27] A. Mannini and A. M. Sabatini, "Machine learning methods for classifying human physical activity from on-body accelerometers," Sensors, vol. 10, no. 2, pp. 1154-1175, 2010.

[28] A. D. Wilson and A. F. Bobick, "Parametric hidden Markov models for gesture recognition," IEEE Transactions on Pattern Analysis and Machine Intelligence, vol. 21, no. 9, pp. 884-900, 1999.

[29] J. Lester, T. Choudhury, N. Kern, G. Borriello, and B. Hannaford, "A hybrid discriminative/generative approach for modeling human activities," in Proceedings of the 19th International Joint Conference on Artificial Intelligence (IJCAI '05), pp. 766-772, 2005.

[30] M. Chen, B. Huang, and Y. Xu, "Human abnormal gait modeling via hidden Markov model," in International Conference on Information Acquisition, pp. 517-522, Seogwipo-si, Jeju-do, korea, July 2007.

[31] C. Zhu, Q. Cheng, and W. Sheng, "Human intention recognition in smart assisted living systems using a hierarchical hidden markov model," in Proceedings of the 4th IEEE International Conference on Automation Science and Engineering, pp. 253-258, Arlington, VA, USA, August 2008.

[32] B. C. Cheng, Y. A. Tsai, G. T. Liao, and E. S. Byeon, "HMM machine learning and inference for activities of daily living recognition," The Journal of Supercomputing, vol. 54, no. 1, pp. 29-42, 2010. 

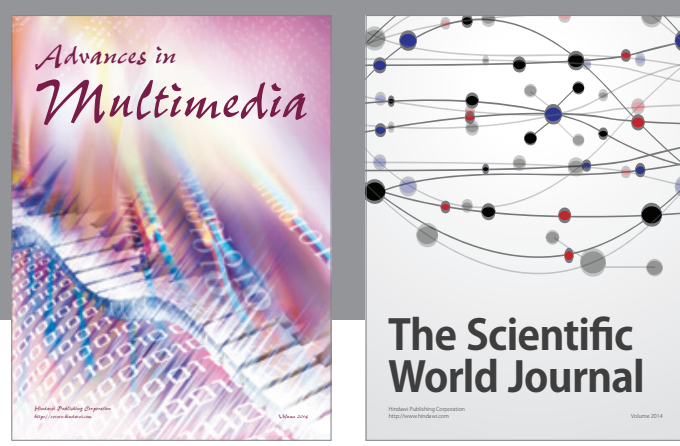

The Scientific World Journal
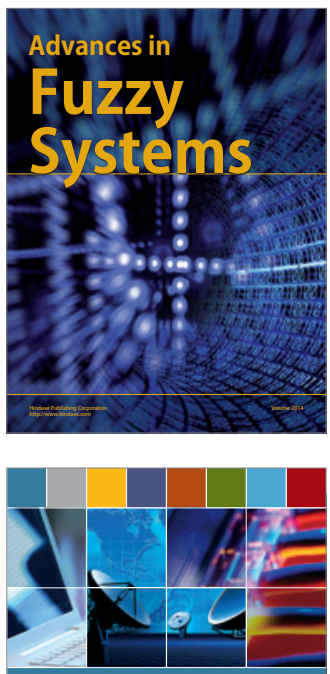

Computer Networks and Communications
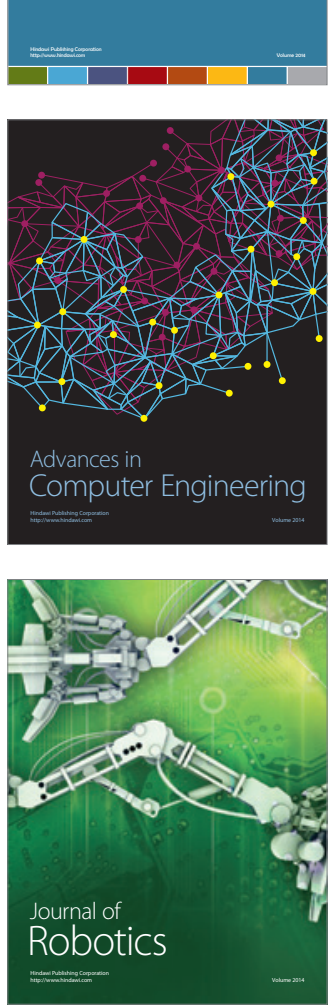
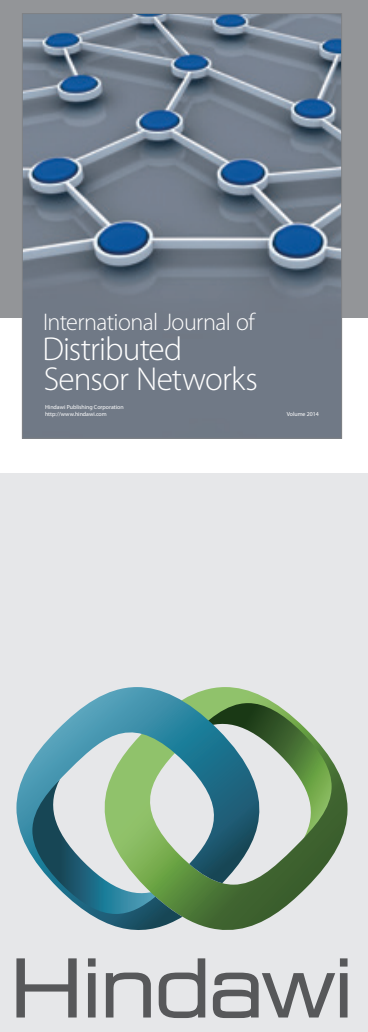

Submit your manuscripts at

http://www.hindawi.com
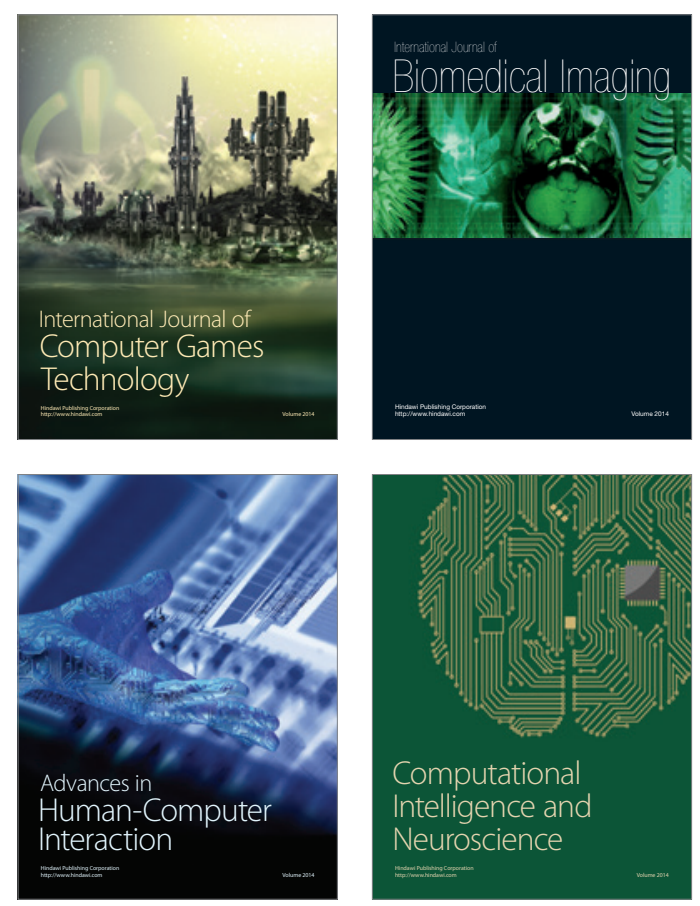
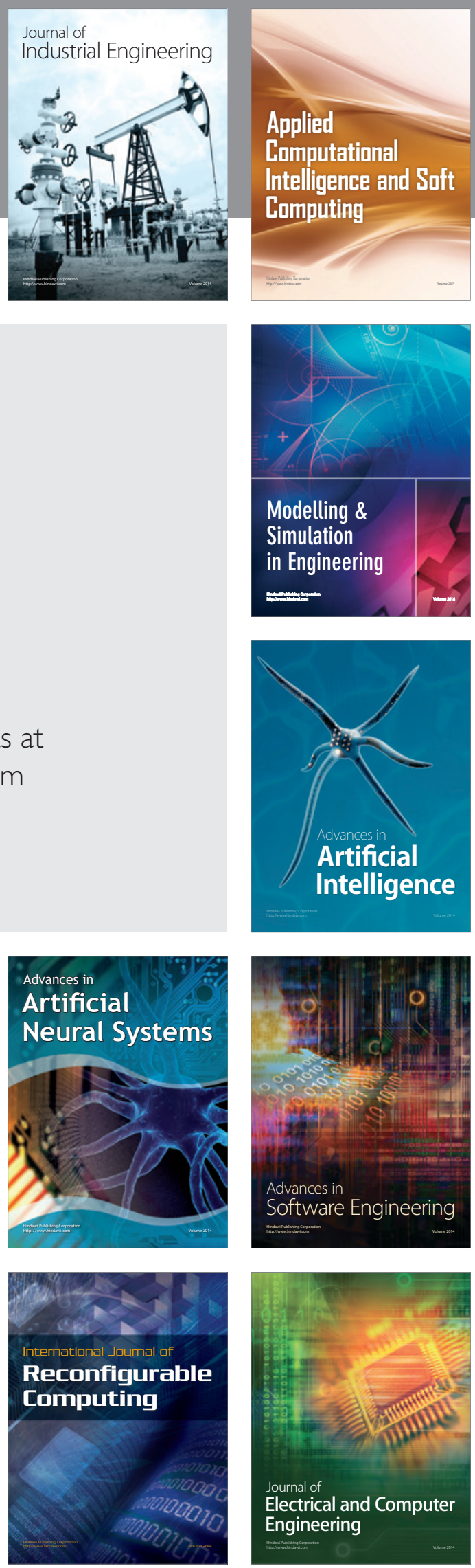